\title{
Spontaneous Spinal Epidural Hematoma: An Atypical Clinical Presentation and Discussion of Management
}

\author{
JASON A. SHAH, BS, ${ }^{1}$ NICK N. PATEL, MD, ${ }^{2}$ KEITH W. MICHAEL, MD ${ }^{2}$ \\ ${ }^{1}$ University of Maryland School of Medicine, Baltimore, Maryland, ${ }^{2}$ Department of Orthopaedic Surgery, Emory University School of Medicine, Atlanta, Georgia
}

\begin{abstract}
Introduction: Spontaneous spinal epidural hematoma (SSEH) is a rare but potentially devastating condition if not appropriately identified and managed. A few case series exist regarding SSEH and certain risk factors have been described; however, much continues to be unknown regarding the pathophysiology and optimal management.

Case Presentation: We present the case of SSEH in a healthy 33-year-old African American woman with no identifiable risk factors who initially presented with significant neurologic compromise. This case reports discusses pertinent clinical presentation, imaging findings, and surgical management. The patient demonstrated near-complete neurologic recovery, highlighting the need for prompt identification and intervention.

Conclusions: We believe this case adds to the limited literature surrounding the topic, particularly in regard to diagnosis and surgical management. It is essential for clinicians to be cognizant of SSEH for timely diagnosis and treatment, even in patients without obvious risk factors.
\end{abstract}

Cervical Spine

Keywords: epidural hematoma, spinal hematoma, cervical spine

\section{INTRODUCTION}

Spontaneous spinal epidural hematoma (SSEH), first described by Jackson $^{1}$ in 1869 , is a rare phenomena with an estimated incidence of 1 in 1000000 per year. ${ }^{2}$ There has been some suggestion of possible association with arteriovenous malformations and coagulopathies; however, little is definitively known on the subject. ${ }^{3-5}$ Multiple case series $^{5-10}$ have demonstrated varying clinical presentations of SSEH with sudden onset back or neck pain, with potential progression to paresthesia and even complete quadriplegia. As a result, SSEH can produce devastating deficits that require immediate medical attention. A few case series have suggested conservative management with appropriate recovery, whereas others have suggested immediate surgical intervention. ${ }^{2,7,11,12} \mathrm{We}$ report a case of SSEH in a young patient with no known predisposing factors and discuss clinical presentation, surgical management, and functional outcomes of the condition.

\section{CASE REPORT}

The patient is a 33-year-old African American woman with no past medical history who presented to the emergency room and reported awakening with acute-onset neck and left arm pain. She also described new-onset paresthesias along with profound weakness in her left upper and lower extremities. The patient experienced a ground-level fall while trying to ambulate due to the significant left leg weakness, which prompted her to present to the emergency room. She denied any preceding trauma and history of medication use including anticoagulants and herbals. She denied any constitutional symptom including fevers, chills, or weight loss. Social history consisted only of occasional alcohol consumption, but she denied any in the preceding days.

On examination, the patient demonstrated tenderness about the left shoulder and paraspinal cervical region. Based on the Medical Research Council's grading system for muscle strength, the patient exhibited global $4 / 5$ muscle strength in the right upper and lower extremities, $2 / 5$ in the left upper extremity, and $1 / 5$ in the left lower extremity. Sensation to light touch was intact in the right upper and lower extremities and intact but globally diminished in the left upper and lower extremities.

A computed tomography (CT) scan of the cervical spine revealed severe spinal canal stenosis 

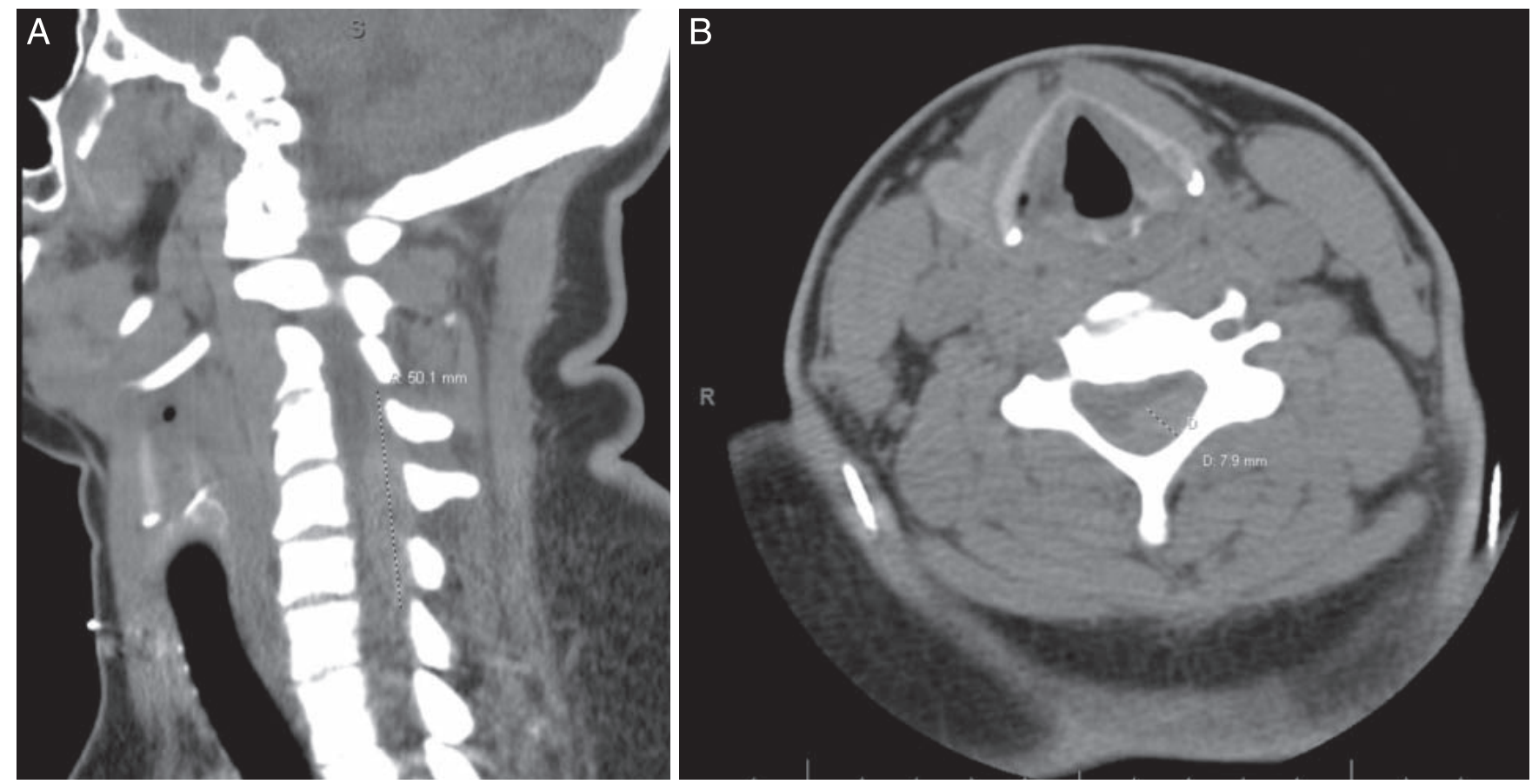

Figure 1. (A) Midline sagittal and (B) C5-C6 axial computed tomography scan images of cervical spine demonstrating left-sided dorsal soft tissue density centered at the C5-C6 level with notable mass effect on the spinal cord.

at the C5-C6 level secondary to a soft tissue density in the dorsal epidural space (Figure 1). Further emergent evaluation with MRI demonstrated a heterogeneous collection within the dorsal epidural space on the left extending from the level of $\mathrm{C} 4$ through C7. This produced severe cord compression with resulting ventral and rightward deviation (Figure 2). Her initial laboratory work-up was within normal limits, including a prothrombin time of 13.1 seconds, international normalized ratio of
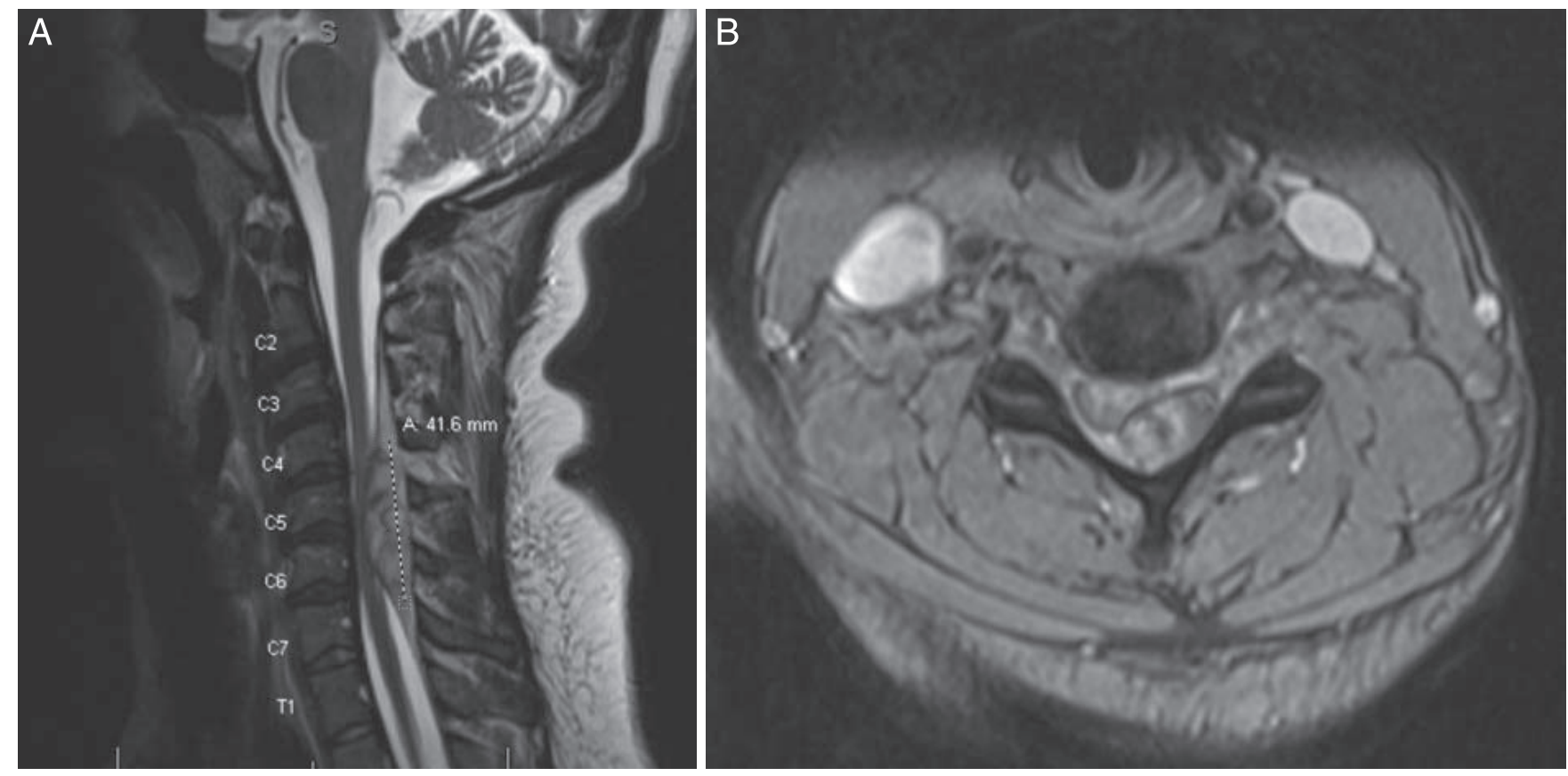

Figure 2. (A) Midline sagittal and (B) C5-C6 axial T2-weighted MRI images without contrast of cervical spine demonstrating heterogeneous left-sided collection within the dorsal epidural space extending from C4 through $\mathrm{C} 7$ and measuring $1.9 \times 1.2 \mathrm{~cm}$ (axial) and $4.2 \mathrm{~cm}$ (craniocaudal). There is severe spinal canal narrowing and resulting cord signal change. 

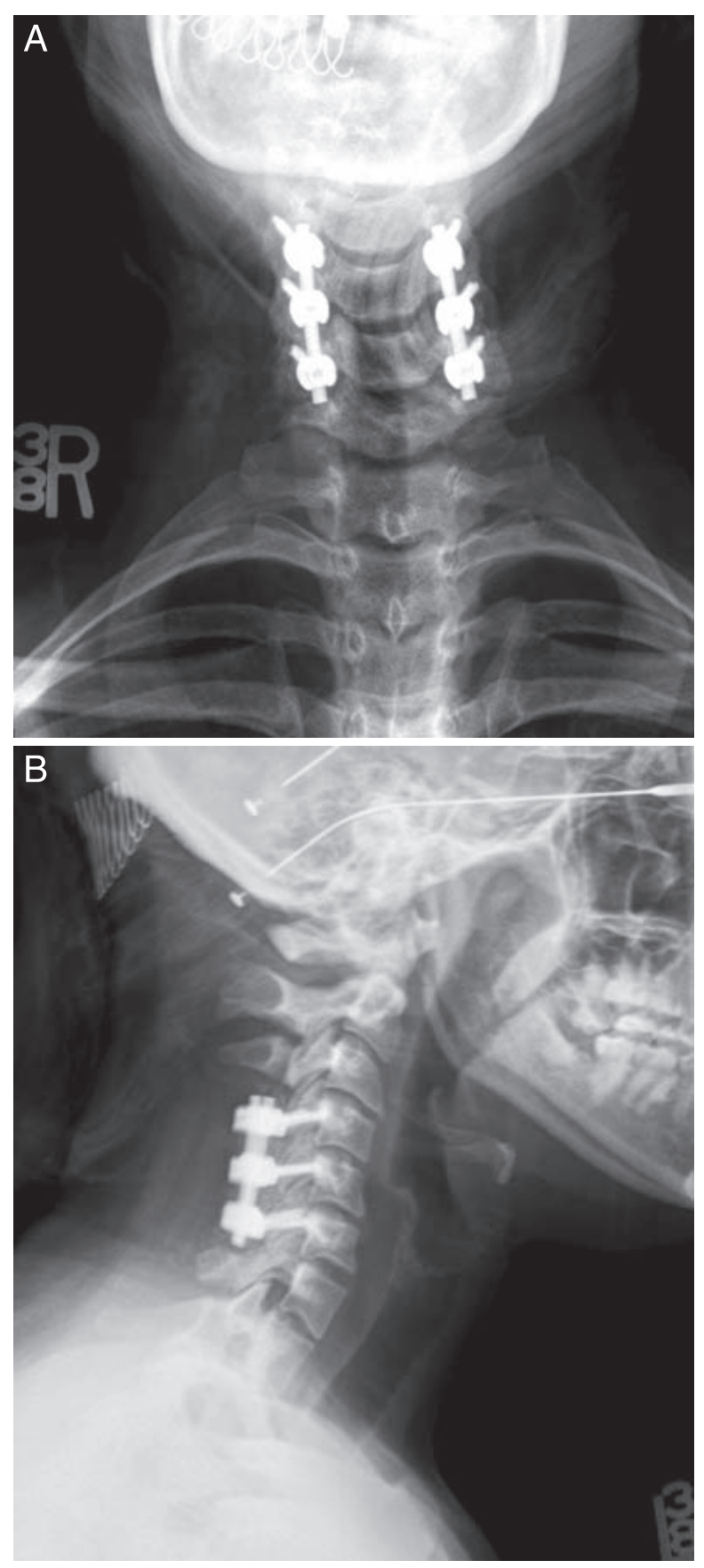

Figure 3. (A) Anteroposterior and (B) lateral upright cervical spine radiographs demonstrating $\mathrm{C} 4$ through $\mathrm{C} 6$ posterior cervical decompression and fusion.

1.2, and platelet count of $177 \times 10^{3} / \mu \mathrm{L}$. Given the patient's acute neurologic deficits in correlation with MRI findings that raised concerns for SSEH, the patient was taken for emergent surgical intervention. A large left-sided, congealed epidural hematoma was encountered that required bilateral $\mathrm{C} 4-\mathrm{C} 6$ laminectomy and C7 dome laminectomy for full evacuation. No vascular malformation or active bleeding source was identified. A C4 through C6 posterior spinal fusion was also performed to minimize the risk of subsequent cervical kyphosis (Figure 3).

The patient demonstrated significant functional improvement through her hospitalization. At time of discharge, 9 days postoperatively, her motor exam was as follows: global $4 / 5$ in right upper and lower extremities, global $4 / 5$ in left upper extremity, and global $3 / 5$ in the left lower extremity. The patient demonstrated return to near baseline at 1 month follow-up with her only deficits being $4 / 5$ motor strength in her left tibialis anterior and extensor hallucis longus.

\section{DISCUSSION}

Spontaneous spinal epidural hematoma is a rare phenomenon that has potential to cause devastating outcomes if not properly diagnosed and managed. Due to its rarity, very little is known about SSEH in regard to pathophysiology and epidemiology. It is generally accepted that most SSEHs are idiopathic in nature and arise from venous epidural plexus; however, literature also supports arterial etiologies. ${ }^{6,9,13}$ The second most common cause is thought to be related to anticoagulant use. ${ }^{14}$ Nevertheless, the topic is still widely debated. To assess for a vascular etiology, our patient did have a CT angiogram in the postoperative period that revealed no obvious vascular anomalies. Another feature unique to SSEH is the challenge in identifying patients who are clinically at risk. Larger metaanalysis demonstrated that the majority of patients are men in the fifth to seventh decade of life. ${ }^{14}$ However, few studies have identified young patients, similar to this presented case, with no previous identifiable risk factors. ${ }^{5,8}$ Therefore, it is essential for physicians to maintain clinical suspicion for this diagnosis in patients who appear demographically at low risk.

In regard to management, our patient was appropriately diagnosed and timely surgical decompression was performed. Surgical decompression and evacuation is generally considered the treatment of choice in a patient diagnosed with SSEH. ${ }^{7,12,14}$ However, there are cases with reported conservative management. ${ }^{2,11}$ Groen $^{11}$ and Holtas et al $^{2}$ reported that a majority of their patients diagnosed with SSEH who were treated nonoperatively recovered completely. However, authors of both studies suggested the success of conservative treatment was most likely secondary to lack of neurologic 
deficits, and limited cord compression was observed in study patients. Our patient presented with symptomology and deficits consistent with severe left-sided cord compression. Although grossly weak on the left side, this patient did have minimal intact motor function preoperatively. This is particularly relevant given that Liao et $\mathrm{al}^{7}$ demonstrated that patients with incomplete neurological deficits preoperatively were statistically more likely to have complete neurological recovery than patients initially presenting with complete deficits. Moreover, Shin et al ${ }^{12}$ demonstrated that patients who received surgical decompression within 24 hours had significantly improved functional recovery compared with patients who had more delayed surgical care. These study findings are consistent with outcomes presented in our case. This patient was quickly diagnosed and surgically decompressed within 9 hours of presentation to the emergency room. At the most recent follow-up, she demonstrated nearcomplete recovery with only minimal deficits in the left leg.

\section{CONCLUSIONS}

Spontaneous spinal epidural hematoma is a rare phenomenon that can produce devastating neurological deficits if not correctly diagnosed and treated. The difficulty arises when attempting to identify a patient population that is clinically at risk. As in this presented case, individuals can present at a young age with no obvious risk factors or laboratory abnormalities. However, it is important to ensure rapid diagnosis and surgical management to provide patients the highest probability of meaningful, functional recovery. This case adds to the limited literature surrounding the topic of SSEH and presents some unique factors associated with patient presentation and management.

\section{REFERENCES}

1. Jackson R. Case of spinal apoplexy. Lancet. 1869;94(2392):5-6.

2. Holtas S, Heiling M, Lonntoft M. Spontaneous spinal epidural hematoma: findings at MR imaging and clinical correlation. Radiology. 1996;199(2):409-413. doi:10.1148/radi ology.199.2.8668786

3. Al-Mutair A, Bednar DA. Spinal epidural hematoma. $J$ Am Acad Orthop Surg. 2010;18(8):494-502.

4. Zhong W, Chen H, You C, Li J, Liu Y, Huang S.
Spontaneous spinal epidural hematoma. J Clin Neurosci. 2011;18(11):1490-1494. doi:10.1016/j.jocn.2011.02.039

5. Figueroa J, DeVine JG. Spontaneous spinal epidural hematoma: literature review. J Spine Surg. 2017;3(1):58-63. doi: $10.21037 /$ jss.2017.02.04

6. Awada A, Russell N, al Fayez N, Naufal R, al Kohlani H. Spontaneous cervical epidural hematoma: case report. Spinal Cord. 1998;36(1):71-72.

7. Liao CC, Lee ST, Hsu WC, Chen LR, Lui TN, Lee SC. Experience in the surgical management of spontaneous spinal epidural hematoma. J Neurosurg. 2004;100(suppl 1, Spine):3845 .

8. Raasck K, Habis AA, Aoude A, et al. Spontaneous spinal epidural hematoma management: a case series and literature review. Spinal Cord Ser Cases. 2017;3:16043. doi:10.1038/ scsandc.2016.43

9. Gopalkrishnan CV, Dhakoji A, Nair S. Spontaneous cervical epidural hematoma of idiopathic etiology: case report and review of literature. J Spinal Cord Med. 2012;35(2):113117. doi:10.1179/2045772312Y.0000000001

10. Baek BS, Hur JW, Kwon KY, Lee HK. Spontaneous spinal epidural hematoma. $J$ Korean Neurosurg Soc. 2008;44(1):40-42. doi:10.3340/jkns.2008.44.1.40

11. Groen RJ. Non-operative treatment of spontaneous spinal epidural hematomas: a review of the literature and a comparison with operative cases. Acta Neurochir (Wien). 2004;146(2):103-110. doi:10.1007/s00701-003-0160-9

12. Shin JJ, Kuh SU, Cho YE. Surgical management of spontaneous spinal epidural hematoma. Eur Spine $J$. 2006;15(6):998-1004. doi:10.1007/s00586-005-0965-8

13. Holtas S, Heiling M, Lonntoft M. Spontaneous spinal epidural hematoma: findings at MR imaging and clinical correlation. Radiology. 1996;199(2):409-413. doi:10.1148/radi ology.199.2.8668786

14. Kreppel D, Antoniadis G, Seeling W. Spinal hematoma: a literature survey with meta-analysis of 613 patients. Neurosurg Rev. 2003;26(1):1-49. doi:10.1007/s10143-002-0224-y

Disclosures and COI: The authors received no funding for this study and report no conflicts of interest.

Corresponding Author: Nick N. Patel, MD, Department of Orthopaedic Surgery, Emory University School of Medicine, 57 Executive Park South, Atlanta, GA 30329. Email: nick.patel@ emory.edu.

Published 30 April 2020

This manuscript is generously published free of charge by ISASS, the International Society for the Advancement of Spine Surgery. Copyright (c) 2020 ISASS. To see more or order reprints or permissions, see http://ijssurgery.com. 